\title{
Research on bus elastic departure interval based on Wavelet Neural Network
}

\author{
Zhe Lv', Jiayu Zou ${ }^{2}{ }^{*}$, and Zhongyu Zhao ${ }^{3}$ \\ ${ }^{1}$ School of Information Engineering, Wuhan University of Technology, 430063 Wuhan, China \\ ${ }^{2}$ School of Information Engineering, Wuhan University of Technology, 430063 Wuhan, China \\ ${ }^{3}$ School of Information Engineering, Wuhan University of Technology, 430063 Wuhan, China
}

\begin{abstract}
In recent years, more and more people choose to travel by bus to save time and economic costs, but the problem of inaccurate bus arrival has become increasingly prominent. The reason is the lack of scientific planning of departure time. This paper takes the passenger flow as an important basis for departure interval, proposes a passenger flow prediction method based on wavelet neural network, and uses intelligent optimization algorithm to study the bus elastic departure interval. In this paper, the wavelet neural network prediction model and the elastic departure interval optimization model are established, and then the model is solved by substituting the data, and finally the theoretical optimal departure interval is obtained.
\end{abstract}

\section{Introduction}

In recent years, with the rapid development of intelligent public transportation system, it is committed to make passengers get more punctual, efficient and consistent public transportation service. A series of achievements have been made in the research of intelligent scheduling of public transport vehicles at home and abroad. Feng Haojie $^{[1]}$ designed an intelligent bus dispatching scheme based on BP neural network and GIS intelligent bus service system; Zhang Tengfei ${ }^{[2]}$ used genetic algorithm to optimize the bus timetable model; Chen Liang ${ }^{[3]}$ et al. Established and solved the bus departure interval calculation model based on the perceived value of passengers on the bus; Meng ziyue ${ }^{[4]}$ and others considered the road congestion and passenger flow demand, established and solved the bus departure interval optimization model; K. Gkiotsalitis ${ }^{[5]}$ uses the cycle optimization method to adjust the bus timetable. In order to make the solution of the bus elastic departure interval more accurate in practice, we can take the passenger flow prediction results as an important basis for decisionmaking.

Lin $\mathrm{Hao}^{[6]}$ and others used spgapso-svm algorithm to predict the bus passenger flow; Huang Yishao ${ }^{[7]}$ proposed a bus passenger flow prediction method based on Rough Set (RS) and improved particle swarm optimization (IPSO) support vector machine (SVM); Yang tianruo ${ }^{[8]}$ established a ternary transfer tensor prediction model to predict the bus passenger flow and invented the corresponding system; Lu Baichuan ${ }^{[9]}$ proposed a shortterm bus passenger flow prediction method based on IC card and RBF neural network; Migensuo ${ }^{[10]}$ and others put forward the combination forecasting model of grey variation particle swarm optimization to predict the bus passenger flow.

With the wide application of neural network prediction model, this paper proposes a method of bus passenger flow prediction based on wavelet neural network, which can significantly improve the prediction accuracy and provide the best data prediction results for the optimization model of departure interval.

\section{Establishment of model}

\subsection{Establishment of wavelet neural network prediction model}

In large cities, there are usually early and late peaks, which makes the fluctuation of data change greatly in a specific period of time, so it is difficult to predict by traditional methods. Therefore, a more flexible wavelet neural network is used to predict the passenger flow data. In this paper, we choose the tight combination prediction model as the basis and Morlet wavelet as the basis function to learn the data. The specific learning steps are as follows:

(1) Forward propagation

$$
h_{j}=h\left(\frac{\sum_{i=1}^{m}\left(\omega_{i j}-b_{j}\right)}{a_{j}}\right),(j=1,2, \ldots, m)
$$

Where, $a_{j}$ is the expansion factor of wavelet basis function, $b_{j}$ is the translation factor of wavelet basis function, and $h(\cdot)$ is Morlet wavelet basis function. The output of wavelet neural network is

* Corresponding author: 214207358@qq.com 


$$
y_{k}=\sum_{j=1}^{m} \omega_{j k} h_{j},(j=1,2, \ldots, m)
$$

The mean square function is chosen as the error function of wavelet neural network, which is

$$
e=\frac{1}{m} \sum_{i=1}^{m}\left(y_{k}-y_{k}^{\prime}\right)^{2}
$$

(2) Back propagation

According to the gradient descent algorithm and error function, the weight and wavelet factor are adjusted continuously. The adjustment formula is as follows

$$
\begin{aligned}
& \omega_{i j}^{(d+1)}=\omega_{i j}^{(d)}+\Delta \omega_{i j}{ }^{(d+1)} \\
& a_{j}^{(d+1)}=a_{j}^{(d)}+\Delta a_{j}^{(d+1)} \\
& b_{j}^{(d+1)}=b_{j}^{(d)}+\Delta b_{j}^{(d+1)}
\end{aligned}
$$

Where, $\mathrm{d}$ is the training times, and $\Delta \omega_{i j}{ }^{(d+1)}$, $\Delta a_{j}{ }^{(d+1)}$ and $\Delta b_{j}{ }^{(d+1)}$ are the adjustment values of the weight and wavelet factor for the $\mathrm{D}+1$ training. The adjustment formula is

$$
\begin{aligned}
\Delta \omega_{i j}{ }^{(d+1)} & =-\eta \frac{\partial e}{\partial \omega_{i j}{ }^{(d)}} \\
\Delta a_{i j}{ }^{(d+1)} & =-\eta \frac{\partial e}{\partial{a_{i j}}^{(d)}} \\
\Delta b_{i j}{ }^{(d+1)} & =-\eta \frac{\partial e}{\partial b_{i j}^{(d)}}
\end{aligned}
$$

Where, $\eta$ represents the learning rate of wavelet neural network and e represents the mean square error function. In order to speed up the convergence rate of wavelet neural network, formula (4) is improved

Where, $\alpha$ is the momentum factor, and $\alpha \in[0,1]$.

\subsection{Establishment of optimization model for elastic departure interval}

Here, the decision variable of the elastic departure model is defined as the departure time $T(i), i=1,2, \ldots, n$ of $\mathrm{N}$ vehicles. According to the reality of life, here we take the upper bound of variable $\mathrm{n}$ as 100 . Secondly, the bus departure time will not be earlier than the first bus departure time $t_{a}$, and will not be later than the last bus departure time $t_{b}$, and the next bus departure time is later than the last bus departure time; Furthermore, it is assumed that the maximum number of passengers $\mathrm{V}$ that the bus can hold is 60; Finally, in order to simplify the problem analysis, this paper only analyzes the situation of passengers getting on and off the bus in one direction.

Assuming that there are a bus stops on the one-way bus line, there are

$$
\Delta W=W_{s, n}-W_{s-1, n}=U_{s, n}-D_{s, n}
$$

Where, $W_{s, n}$ is the number of people on the bus after the nth bus passes the s station, $\Delta W$ is the number of people on the bus, $U_{s, n}$ is the number of people getting on the bus at the station, and $D_{s, n}$ is the number of people getting off the bus at the station.

The number of passengers will be limited by the waiting number of the current station and the bus capacity

$$
q_{s, n+1}=q_{s, n}-U_{s, n}+\int \lambda(t) d t
$$

Where, $\lambda(t)$ is the number of people who arrive at the bus stop in a period of time. For waiting passengers, the waiting time can be calculated as

$$
T_{w}=\sum_{i=1}^{n} \sum_{s=1}^{A} \frac{1}{2}\left(q_{s, n-1}-U_{s, n-1}+q_{s, n}\right)[T(n)-T(n-1)]
$$

Considering that the cost of bus operation can't be higher than a certain threshold, there are some problems

$$
C=\sum_{i=1}^{n} c_{i} f_{2} S t^{\prime}-f_{1} \int_{t_{b}}^{t_{a}} \lambda(t) d t \leq K_{0}
$$

Where, $f_{1}$ is the ticket price and $f_{2}$ is the bus cost per unit time. According to the related literature, the threshold $\mathrm{K}_{0}$ is 785 .

Finally, the total objective function is obtained

$$
\min =\omega_{1} T_{w} \delta+\omega_{2} C
$$

Where, $\delta$ is the unit time value technology. According to the relevant literature, $\delta$ is taken as 8.6.

Finally, the total model is obtained as follows

$$
\begin{gathered}
\min =\omega_{1} T_{w} \delta+\omega_{2} C \quad \\
n<100 \\
t_{a} \leq T(n) \leq t_{b} \\
T(i) \leq T(i+1) \\
V=60 \\
T_{w}=\sum_{i=1}^{n} \sum_{s=1}^{A} \frac{1}{2}\left(\begin{array}{c}
\left.q_{s, n-1}-U_{s, n-1}+q_{s, n}\right) \\
C \leq K_{0} \\
U_{s, n}=\int_{t(n-1)+(s-1) t^{\prime}}^{t(n)+(s-1) t^{\prime}}
\end{array} \lambda(t) d t\right.
\end{gathered}
$$

\subsection{Model solving}

The data of Wuhan passenger flow is preprocessed and divided into training set and test set with the ratio of 8:2. At the same time, the parameters are initialized, the training set is substituted into the model, and the gradient descent algorithm is used to optimize the parameters. Finally, the trained model is used to predict the data of the test set, and the passenger flow prediction chart can be obtained, as shown in Figure 1. The error comparison 
chart is shown in Figure 2.

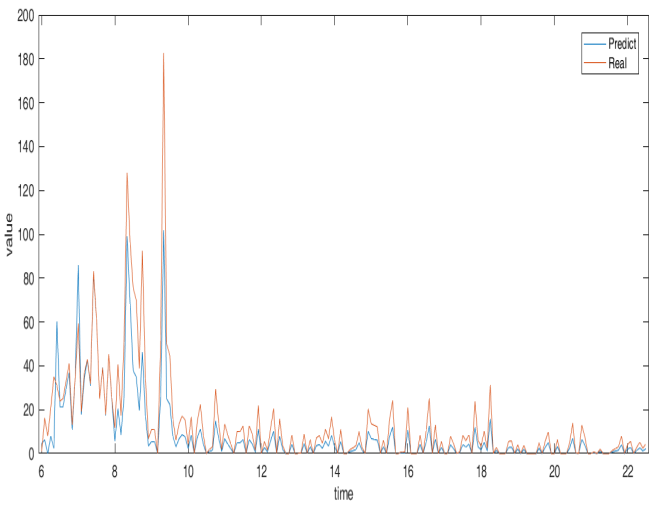

Figure 1 passenger flow forecast

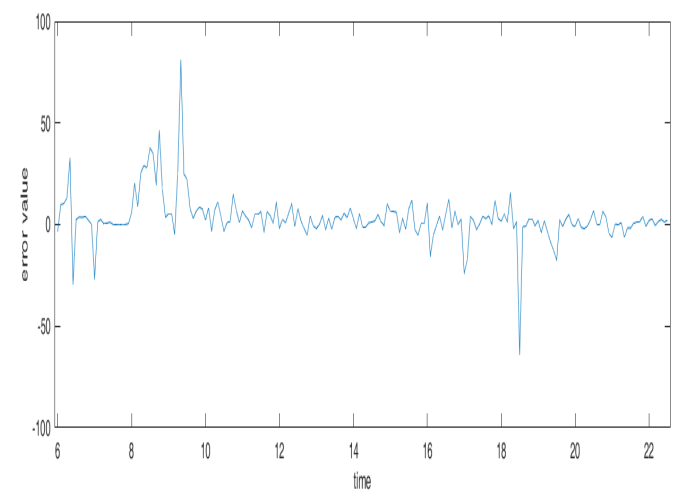

Figure 2 error analysis chart

According to Figure 1 and Figure 2, it can be considered that the prediction accuracy of wavelet neural network for passenger flow is higher, the prediction error is smaller, and the prediction result is more reliable.

Using the data predicted by wavelet neural network and gray wolf optimization algorithm, the elastic departure interval optimization model is solved, and the actual departure time scheme can be obtained, as shown in Table 1.

Table 1 departure time scheme

\begin{tabular}{|l|l|l|l|}
\hline $\begin{array}{l}\text { Departure } \\
\text { time }\end{array}$ & $\begin{array}{l}\text { Departure } \\
\text { time }\end{array}$ & $\begin{array}{l}\text { Departure } \\
\text { time }\end{array}$ & $\begin{array}{l}\text { Departure } \\
\text { time }\end{array}$ \\
\hline $2020 / 6 / 9$ & $2020 / 6 / 9$ & $2020 / 6 / 9$ & $2020 / 6 / 9$ \\
$6: 25$ & $8: 35$ & $12: 45$ & $17: 35$ \\
\hline $2020 / 6 / 9$ & $2020 / 6 / 9$ & $2020 / 6 / 9$ & $2020 / 6 / 9$ \\
$6: 30$ & $8: 50$ & $13: 05$ & $17: 55$ \\
\hline $2020 / 6 / 9$ & $2020 / 6 / 9$ & $2020 / 6 / 9$ & $2020 / 6 / 9$ \\
$6: 35$ & $9: 00$ & $13: 25$ & $18: 15$ \\
\hline $2020 / 6 / 9$ & $2020 / 6 / 9$ & $2020 / 6 / 9$ & $2020 / 6 / 9$ \\
$6: 40$ & $9: 10$ & $13: 50$ & $18: 35$ \\
\hline $2020 / 6 / 9$ & $2020 / 6 / 9$ & $2020 / 6 / 9$ & $2020 / 6 / 9$ \\
$6: 45$ & $9: 25$ & $14: 15$ & $18: 55$ \\
\hline $2020 / 6 / 9$ & $2020 / 6 / 9$ & $2020 / 6 / 9$ & $2020 / 6 / 9$ \\
$6: 55$ & $9: 40$ & $14: 40$ & $19: 10$ \\
\hline $2020 / 6 / 9$ & $2020 / 6 / 9$ & $2020 / 6 / 9$ & $2020 / 6 / 9$ \\
$7: 05$ & $10: 00$ & $15: 00$ & $19: 25$ \\
\hline $2020 / 6 / 9$ & $2020 / 6 / 9$ & $2020 / 6 / 9$ & $2020 / 6 / 9$ \\
$7: 15$ & $10: 20$ & $15: 20$ & $20: 45$ \\
\hline $2020 / 6 / 9$ & $2020 / 6 / 9$ & $2020 / 6 / 9$ & $2020 / 6 / 9$ \\
$7: 25$ & $10: 40$ & $15: 40$ & $21: 05$ \\
\hline
\end{tabular}

\begin{tabular}{|l|l|l|l|}
\hline $2020 / 6 / 9$ & $2020 / 6 / 9$ & $2020 / 6 / 9$ & $2020 / 6 / 9$ \\
$7: 35$ & $11: 00$ & $15: 55$ & $21: 20$ \\
\hline $2020 / 6 / 9$ & $2020 / 6 / 9$ & $2020 / 6 / 9$ & $2020 / 6 / 9$ \\
$7: 45$ & $11: 15$ & $16: 10$ & $21: 35$ \\
\hline $2020 / 6 / 9$ & $2020 / 6 / 9$ & $2020 / 6 / 9$ & $2020 / 6 / 9$ \\
$7: 55$ & $11: 30$ & $16: 25$ & $21: 55$ \\
\hline $2020 / 6 / 9$ & $2020 / 6 / 9$ & $2020 / 6 / 9$ & $2020 / 6 / 9$ \\
$8: 05$ & $11: 45$ & $16: 45$ & $22: 15$ \\
\hline $2020 / 6 / 9$ & $2020 / 6 / 9$ & $2020 / 6 / 9$ & \\
$8: 15$ & $12: 00$ & $17: 05$ & \\
\hline $2020 / 6 / 9$ & $2020 / 6 / 9$ & $2020 / 6 / 9$ & \\
$8: 25$ & $12: 20$ & $17: 20$ & \\
\hline
\end{tabular}

Substituting the departure time scheme into the bus status network, the arrival time table can be obtained, as shown in table 2.

Table 2 departure status

\begin{tabular}{|c|c|c|c|}
\hline $6: 25$ & $6: 30$ & $\ldots$ & $7: 15$ \\
\hline $6: 30$ & $6: 35$ & $\ldots$ & $7: 20$ \\
\hline $6: 35$ & $6: 40$ & $\ldots$ & $7: 25$ \\
\hline $6: 40$ & $6: 45$ & $\ldots$ & $7: 30$ \\
\hline $6: 45$ & $6: 50$ & $\ldots$ & $7: 35$ \\
\hline $6: 55$ & $7: 00$ & $\ldots$ & $7: 45$ \\
\hline$\ldots$ & $\ldots$ & & $\ldots$ \\
\hline $22: 15$ & $22: 20$ & $\ldots$ & $23: 10$ \\
\hline
\end{tabular}

Through the solution of the final joint model, the number of departure of buses at different times can be inferred according to the actual traffic volume, and then the schedule of departure status of buses can be obtained. According to the time schedule of departure status and the edge cloud equipment, the departure time of buses can be arranged adaptively according to the traffic and traffic flow detected by the edge equipment, so as to save resources, alleviate traffic conditions and improve traffic efficiency. Through the actual operation test of the bus in Wuhan, the accuracy of bus arrival was improved to $98.86 \%$ before and after the implementation of the strategy.

\section{Conclusion}

In this paper, the wavelet neural network is used to train and predict the passenger flow data of Wuhan City, and the intelligent optimization algorithm is used to optimize the departure interval model. Finally, the optimal bus departure time can be reasonably planned, which can save a lot of resources and alleviate the traffic congestion to a certain extent. In this paper, neural network and intelligent optimization algorithm are applied to complex traffic network flexibly, and good results are obtained. In the process of the gradual development of the intelligent transportation field, the methods of deep learning and machine vision can be used to implement better intelligent traffic control, which will further save resources and alleviate the traffic situation.

\section{Reference}

1. Feng Haojie. Design of intelligent public 
transportation service system based on BP neural network and GIS [D]. Henan: Henan University of technology, 2018:45-48

2. Zhang Tengfei. Optimization of bus departure schedule based on unbalanced passenger flow [D]. Liaoning: Dalian Jiaotong University, 2019:37-45

3. Chen Liang, Feng Liu, Li qiaoru. Calculation model of bus departure interval considering the perceived value of in car $[\mathrm{J}]$. Journal of Harbin Institute of technology, 2018,50 (03): 150-155

4. Meng ziyue, Zuo Zhongyi, Guo Runhang. Optimization of bus departure interval considering road congestion and passenger flow demand $[\mathrm{J}]$. Journal of Dalian Jiaotong University, 2019,40 (04): $13-17+33$

5. K. Gkiotsalitis. A model for the periodic optimization of bus dispatching times[J]. Applied Mathematical Modelling,2020,82.

6. Lin Hao, Li Leixiao, Wang Hui. Spgapso-svm: an algorithm for urban public transport passenger flow prediction [J]. Minicomputer system, 2020,41 (11): 2458-2464

7. Huang Yishao, Han Lei. Prediction method of bus passenger flow based on rs-ipsosvm [J]. Journal of Chongqing Jiaotong University (NATURAL SCIENCE EDITION), 2020,39 (11): 11-19

8. Yang tianruo, Zhang Shunli, Liao Yang. A prediction method and system for bus passenger flow: China, $201810439786.5[\mathrm{P}]$. August 17, 2018 